\title{
Hepatoprotective effect of the flavonoid fraction isolated from the flower of Inula britannica against D-Galactosamine-induced hepatic injury
}

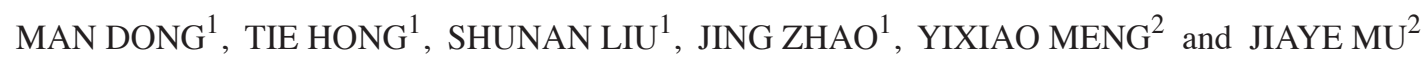 \\ ${ }^{1}$ Department of Pharmacology, School of Pharmacy, Jilin University, Changchun 130021; \\ ${ }^{2}$ Yanbian University Health Science Center, Yanji 133002, P.R. China
}

Received January 8, 2013; Accepted March 21, 2013

DOI: $10.3892 / \mathrm{mmr} .2013 .1443$

\begin{abstract}
The aim of this study was to investigate the mechanism and nature of the protective effect of Inula britannica flower flavonoids (IBFF) on antioxidants and the inhibition of inflammation in liver injury. Liver injury was induced in a mouse model by intraperitoneal injection of D-Galactosamine (D-Gal; $850 \mathrm{mg} / \mathrm{kg}$ ) and IBFF was administered orally at 125 , 250 or $500 \mathrm{mg} / \mathrm{kg}$ once a day for 7 days. The results revealed that IBFF reversed the increases in serum aminotransferase levels and lipid peroxidation and also reversed the decreases in hepatic glutathione content. IBFF attenuated the D-Galinduced increases in tumornecrosis factor- $\alpha$ (TNF- $\alpha)$, inducible nitric oxide synthase (iNOS) and cyclooxygenase-2 (COX-2) mRNA and protein levels in the liver. Our data suggest that IBFF ameliorates D-Gal-induced acute liver injury and that this protection may be due to its antioxidative and anti-inflammatory activities.
\end{abstract}

\section{Introduction}

Liver disease is one of the most serious health problems worldwide, yet despite tremendous advances in modern medicine, prevention and treatment options remain limited. The pathogenesis of hepatic diseases, as well as the role of oxidative stress and inflammation, is well established (1). Therefore, blocking or retarding the chain reactions involved in these oxidation and inflammatory processes is a promising therapeutic strategy for the prevention and treatment of liver injury.

There is increasing evidence that the subcutaneous injection of high doses of D-Galactosamine (D-Gal) induces

Correspondence to: Professor Tie Hong, Department of Pharmacology, School of Pharmacy, Jilin University, No. 1266 Fujin Road, Changchun, Jilin 130021, P.R. China

E-mail: hongtie@jlu.edu.cn

Key words: Inula britannica flower flavonoids, D-Galactosamine, oxidative stress, inflammatory response, mouse liver injury severe oxidative stress, as indicated by elevated levels of malonaldehyde (MDA) and decreased levels of antioxidant enzymes in a murine model $(2,3,4)$. D-Gal-induced liver injury is a well-established experimental model that closely resembles the morphological and functional features of human hepatitis. This model has provided insights into the pathogenesis of clinical hepatitis that are useful for developing novel liver-protective reagents.

The Inula britannica flower (IBF) is well known in traditionalChinese herbal medicine. Numerous studies have reported that the IBF has multiple biological and pharmacological effects, including antitumor, antidiabetic $(5,6,7)$, immunomodulatory (8) and hepatoprotective functions $(9,10,11)$. However, the mechanism by which IBF flavonoids (IBFF) exert their hepatoprotective effect has not yet been reported.

The aim of this study was to explore whether IBFF protects the mouse liver from D-Gal-induced injury by attenuating oxidative stress and suppressing the inflammatory response.

\section{Materials and methods}

Materials and reagents. The IBF was obtained from Jishen Pharmacy (Changchun, China). D-Gal was obtained from Qidong Jiufeng Industry and Trade Co., Ltd. (Jiangsu, China). Alanine aminotransferase (ALT), aspartate aminotransferase (AST), superoxide dismutases (SODs), MDA, glutathione peroxidase (GSH-PX), catalase (CAT) and inducible nitric oxide synthase (iNOS) kits were obtained from Nanjing Jiancheng Institute of Biotechnology (Nanjing, China). ELISA kits were obtained from R\&D Systems (Shanghai, China). The real time-PCR (RT-PCR) kit was obtained from Takara Biotechnology (Dalian) Co., Ltd (Shiga, Japan).

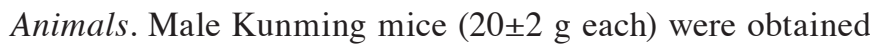
from the Jilin University Laboratory Animal Center (Changchun, China). The mice were maintained under a constant 12 -h light and dark cycle at an environmental temperature of $25^{\circ} \mathrm{C}$ and $45-55 \%$ relative humidity. All experiments were performed according to the Guidelines of the Experimental Laboratory Animal Committee of the Jilin Province. The study was approved by the ethics committee of Jilin university, Changchun, China. 
Preparation of IBFF. The IBF was soaked in $85 \%$ ethanol solvent $(1: 20 \mathrm{w} / \mathrm{v})$ for $3 \mathrm{~h}$, placed in an ultrasonic bath and sonicated at $200 \mathrm{kHz}$ at $55^{\circ} \mathrm{C}$ for $30 \mathrm{~min}$. The samples were filtered through a $0.45 \mu \mathrm{m}$ microporous membrane (Shanghai Wanzi Shiye Co., Ltd., Shanghai, China). The IBF was extracted two additional times with the same volume of fresh solvent and the filtrate was collected. The filtrates were mixed and concentrated under reduced pressure in a rotary evaporator to yield dried crude extracts. Distilled water was added to the extracts, which were then defatted using petroleum ether and ethyl acetate. The total flavonoid extracts were purified using AB-8 resin under the following conditions: the extracted sample concentration was $20 \mathrm{mg} / \mathrm{ml}$ and washing was performed with $70 \%$ ethanol at a flow velocity of $2 \mathrm{ml} / \mathrm{min}$.

The total flavonoid content of the extracts was determined by the method described in the Chinese Pharmacopoeia (2005, p211-212). The extracts $(500 \mu \mathrm{l})$ were diluted and mixed with $1 \mathrm{ml} 5 \% \mathrm{NaNO}_{2}$. After standing for $6 \mathrm{~min}, 1 \mathrm{ml} 10 \% \mathrm{AlCl}_{3}$ and $10 \mathrm{ml} \mathrm{NaOH}(1 \mathrm{M})$ were added to the mixture. The mixture was adjusted to $25 \mathrm{ml}$ by adding $70 \%$ ethanol and allowed to rest for $15 \mathrm{~min}$. Absorbance (A) was measured at $510 \mathrm{~nm}$ with $70 \%$ ethanol as a blank control. Rutin was used as a reference standard and the total flavonoid content was expressed relative to rutin. The result revealed that the IBFF content of an IBF was $86.54 \%$.

Animal grouping and treatment. The mice were divided into 5 groups (10 mice in each group) as follows: the control, D-Gal-treated, low-dose (IBFF $125 \mathrm{mg} / \mathrm{kg}$ body weight plus D-Gal), middle-dose (IBFF $250 \mathrm{mg} / \mathrm{kg}$ body weight plus D-Gal) and high-dose groups (IBFF $500 \mathrm{mg} / \mathrm{kg}$ body weight plus D-Gal). All IBFF groups received the indicated concentration of IBFF orally every $24 \mathrm{~h}$ for 7 consecutive days. To evaluate the effects of IBFF in the liver injury mouse model, D-Gal was intraperitoneally injected once at a dose of $850 \mathrm{mg} / \mathrm{kg}$ on day $7,1 \mathrm{~h}$ after oral administration of IBFF. An equal volume of saline instead of D-Gal was injected into the mice of the control group.

Determination of liver enzyme activity. Liver injury was assessed $24 \mathrm{~h}$ after D-Gal injection by measuring the enzyme levels of ALT and AST in the serum. The levels of ALT and AST were expressed as an international unit (U/l). Liver enzyme levels were determined according to the manufacturer's instructions for the ALT and AST kits.

Measurement of lipid peroxidation level. The lipid peroxidation level of MDA was determined by kits from Nanjing Jiancheng Bioengineering Institute (Nanjing, China). All procedures were performed according to the manufacturer's instructions. MDA in the liver was determined by measuring the level of thiobarbituric acid-reactive substances spectrophotometrically at $535 \mathrm{~nm}$.

Measurement of antioxidant enzyme activity. The activity of the antioxidant enzymes SOD, GSH-PX and CAT were determined by kits obtained from Nanjing Jiancheng Bioengineering Institute. All procedures were performed according to the manufacturer's instructions. SOD activity in liver homogenates was detected using the xanthine oxidase method. The levels

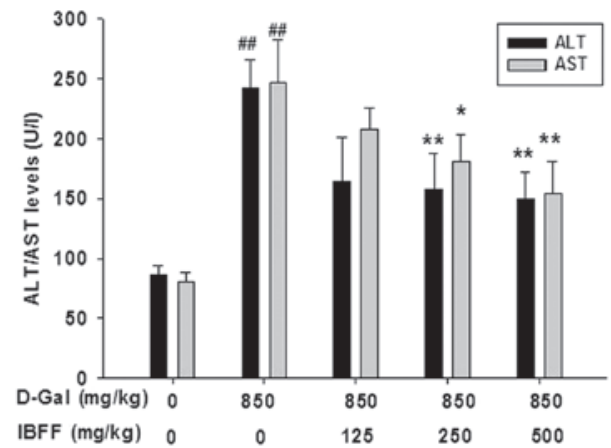

Figure 1. Effects of IBFF on the serum ALT and AST levels in D-Gal-induced liver injury. The values reported are the means $\pm \mathrm{SE}$. ${ }^{\# \#} \mathrm{P}<0.01$ compared with the control group; ${ }^{* *} \mathrm{P}<0.01,{ }^{*} \mathrm{P}<0.05$ compared with the model group. D-Gal, D-Galactosamine; IBFF, Inula britannica flower flavonoids; ALT, alanine aminotransferase; AST, aspartate aminotransferase.

of GSH-PX in the liver homogenates were determined using a colorimetric dithiobis(2-nitrobenzoic acid) assay. CAT activity was assayed by measuring the decomposition of $\mathrm{H}_{2} \mathrm{O}_{2}$.

Measurement of serum TNF- $\alpha, C O X-2$ and iNOS levels. The serum TNF- $\alpha$ and COX-2 levels were measured using ELISA kits (R\&D Systems) according to the manufacturer's instructions. The iNOS level was determined by a kit from the Nanjing Jiancheng Bioengineering Institute. iNOS activity was determined by measuring the level of NO generated by sodium nitroprusside.

Histological evaluation. The livers were perfused transcardially with $25 \mathrm{ml}$ of normal saline $(0.9 \%)$. Formalin-fixed specimens were embedded in paraffin and HE-stained and images were captured under a light microscope. The liver morphology was assessed based on the following parameters: vacuolization, nuclear condensation, lymphocyte infiltration and erythrocyte stasis.

RT-PCR analysis. Total RNA was isolated from liver tissues using TRIzol reagent. First-strand complementary DNA (cDNA) was synthesized using oligo(dT) primers and M-MLV reverse transcriptase. RT-PCR was performed using PCR Master Mix. The following primers were used to amplify TNF- $\alpha$, COX-2 and iNOS: TNF- $\alpha$, sense 5'-TCTCAAGTC TCCACAAGAGG-3' and antisense 5'-TGAGTTGTAACC AGGTCAG-3'; COX-2, sense 5'-AGACATCCTGATCCT GGTTT-3' and antisense 5'-GTTCAATGGGCTGGAAG ACA-3'; iNOS, sense 5'-GCTCGGGTTGAAGTGGTAT-3' and antisense 5'-TGAAGGACTCTGAGGCTGT-3'; and $\beta$-actin, sense 5'-ATATCGCTGCGCTGGTCGTC-3' and antisense 5'-AGGATGGCGTGAGGGAGAGC-3'. The TNF- $\alpha$ PCR conditions were as follows: denaturation at $94^{\circ} \mathrm{C}$ for $30 \mathrm{sec}$, annealing at $52^{\circ} \mathrm{C}$ for $30 \mathrm{sec}$ and elongation at $72^{\circ} \mathrm{C}$ for $60 \mathrm{sec}$. For COX-2 and iNOS, the PCR conditions were as follows: denaturation at $94^{\circ} \mathrm{C}$ for $30 \mathrm{sec}$, annealing at $53^{\circ} \mathrm{C}$ for $30 \mathrm{sec}$ and elongation at $72^{\circ} \mathrm{C}$ for $60 \mathrm{sec} . \beta$-actin was used as a reference gene to normalize the expression level of each gene.

Statistics. The data are expressed as the mean \pm SE. The statistical significance of the differences detected for each parameter 


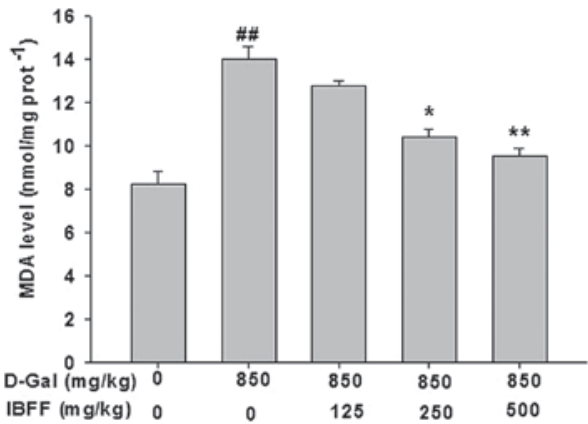

Figure 2. Effect of IBFF on the lipid peroxidation level in D-Gal-induced liver injury. The values are reported as the means $\pm \mathrm{SE}$. ${ }^{\# \#} \mathrm{P}<0.01 \mathrm{com}-$ pared with the control group; ${ }^{* *} \mathrm{P}<0.01,{ }^{*} \mathrm{P}<0.05$ compared with the model group. D-Gal, D-Galactosamine; IBFF, Inula britannica flower flavonoids; MDA, malondialdehyde.

$\mathbf{A}$

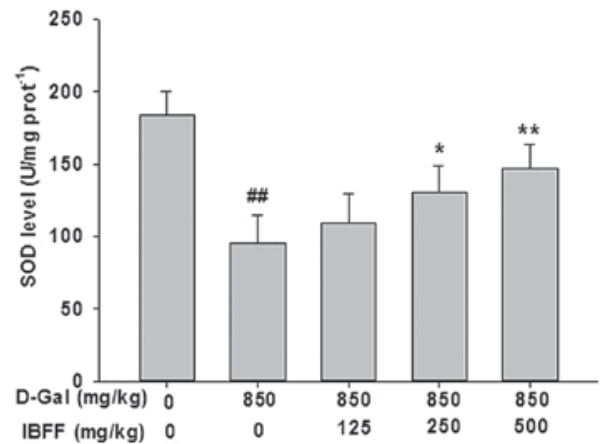

B

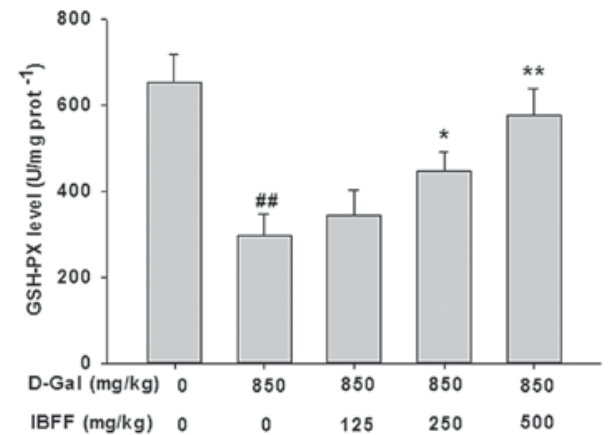

C

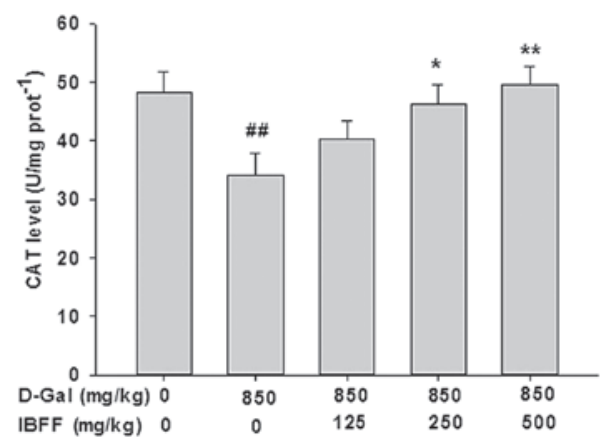

Figure 3. (A) Effect of IBFF on SOD levels in D-Gal-induced liver injury. The SOD level of the model group decreased compared with the control group $\left({ }^{\# \#} \mathrm{P}<0.01\right)$, while the SOD levels increased significantly in the IBFF-treated groups ( ${ }^{* *} \mathrm{P}<0.01,{ }^{*} \mathrm{P}<0.05$ ). (B) Effect of IBFF on the GSH-PX level in D-Gal-induced liver injury. The GSH-PX level of the model group increased compared with that of the control group $\left({ }^{\# \#} \mathrm{P}<0.01\right)$ and GSH-PX levels increased in the IBFF-treated groups $\left({ }^{* *} \mathrm{P}<0.01,{ }^{*} \mathrm{P}<0.05\right)$. (C) Effect of IBFF on the CAT level in D-Gal-induced liver injury. Values are reported as the means \pm SE. ${ }^{\# /} \mathrm{P}<0.01$ compared with the control group; ${ }^{* *} \mathrm{P}<0.01,{ }^{*} \mathrm{P}<0.05$ compared with the model group. D-Gal, D-Galactosamine; IBFF, Inula britannica flower flavonoids; SOD, superoxide dismutase; GSH-PX, glutathione peroxidase; CAT, catalase.
A

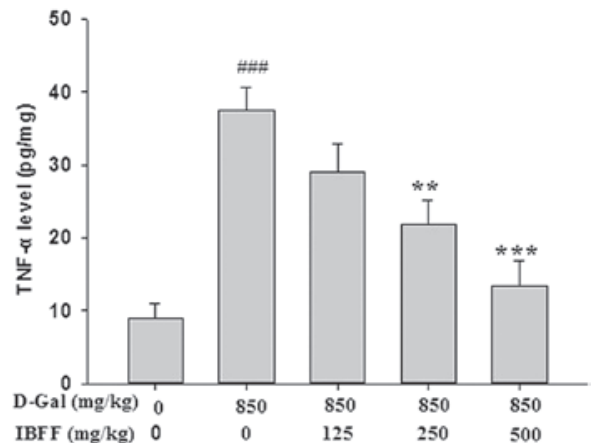

B

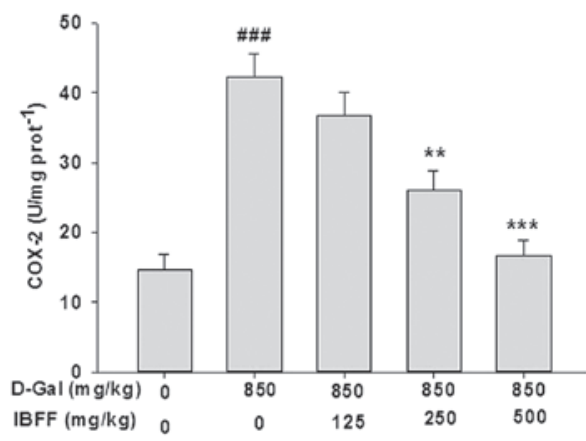

C

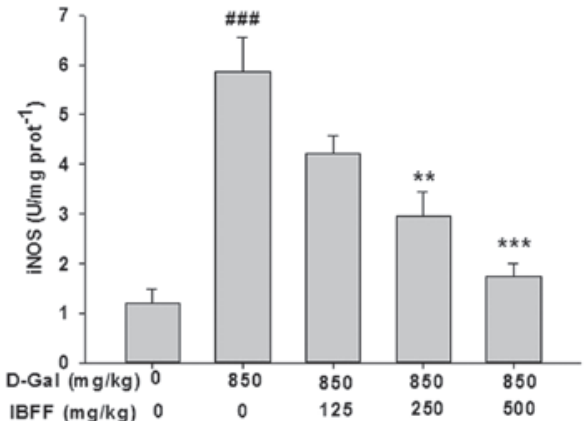

Figure 4. (A) Effect of IBFF on the TNF- $\alpha$ level in D-Gal-induced liver injury. The values are reported as the mean $\pm \mathrm{SE}$. ${ }^{\# \#} \mathrm{P}<0.001$ compared with the control group; ${ }^{* *} \mathrm{P}<0.01,{ }^{* * * *} \mathrm{P}<0.001$ compared with the model group. (B) Effect of IBFF on the COX-2 level in D-Gal-induced liver injury. Values are reported as the means $\pm \mathrm{SE},{ }^{\# \# \#} \mathrm{P}<0.001$ compared with the control group; ${ }^{* *} \mathrm{P}<0.01$, ${ }^{* * * *} \mathrm{P}<0.001$ compared with the model group. (C) Effect of IBFF on the iNOS level in D-Gal-induced liver injury. Values are reported as the means $\pm \mathrm{SE}$, ${ }^{\# \# \#} \mathrm{P}<0.01$ compared with the control group; ${ }^{* *} \mathrm{P}<0.01,{ }^{* * *} \mathrm{P}<0.001$ compared with the model group. D-Gal, D-Galactosamine; IBFF, Inula britannica flower flavonoids; TNF- $\alpha$,tumor necrosis factor- $\alpha$; COX-2, cyclooxygenase- 2 ; iNOS, inducible nitric oxide synthase.

among the groups was evaluated using one-way analysis of variance (ANOVA), followed by Fisher's protected least significantdifference(PLSD)comparisontestforposthoct-tests.

\section{Results}

Effects of IBFF on serum ALT and AST levels inD-Gal-induced liver injury. Several hepatic enzymes, including AST and ALT in the serum were used as biochemical markers for acute hepatic damage. The levels of AST and ALT were measured in the serum to evaluate hepatic tissue damage. D-Gal administration resulted in a statistically significant $(\mathrm{P}<0.01)$ increase in the AST and ALT levels compared with the control group. Groups treated with IBFF $(125,250,500 \mathrm{mg} / \mathrm{kg})$ did not show an increase in the levels of AST and ALT in the serum (Fig. 1). 
A

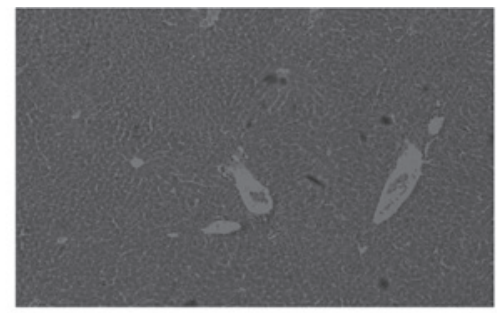

B

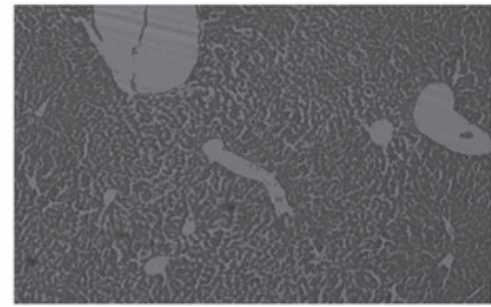

C

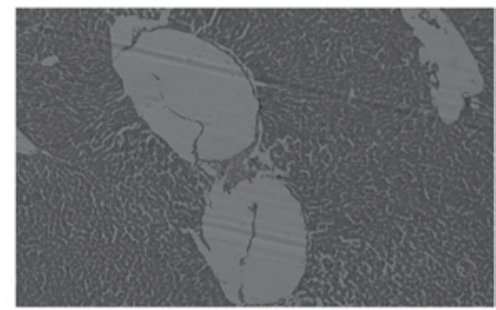

D

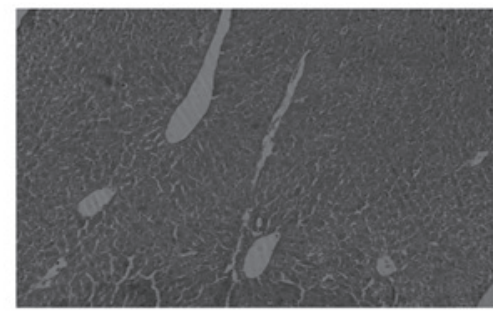

$\mathbf{E}$

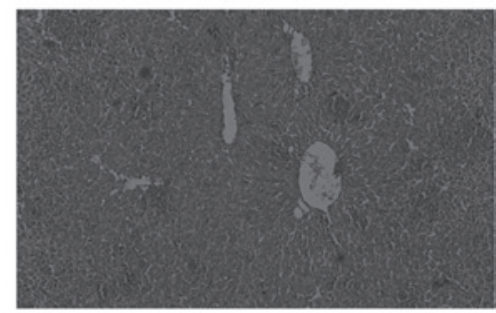

Figure 5. Effects of IBFF on the histopathological changes of D-Gal-induced acute liver injury. (A) Liver morphology in the control group with normal lobular architecture and cell structure. (B) Liver structure in the model group with extensive hepatocellular damage and the presence of portal inflammation and central zone necrosis. (C) Liver morphology in the $125 \mathrm{mg} / \mathrm{kg}$ IBFF-treated group. (D) Liver morphology in the $250 \mathrm{mg} / \mathrm{kg}$ IBFF-treated group. (E) Liver morphology in the $500 \mathrm{mg} / \mathrm{kg}$ IBFF-treated group. Magnification, $\mathrm{x} 100$. D-Gal, D-Galactosamine; IBFF, Inula britannica flower flavonoids.

Effect of IBFF on the lipid peroxidation level in D-Gal-induced liver injury. Hepatic levels of MDA were assessed as an indicator of liver tissue lipid peroxidation. An increase $(\mathrm{P}<0.01)$ in the levels of MDA was observed in the D-Gal-treated group when compared with the control group. The D-Gal-induced elevation of the tissue MDA concentration was reduced $(\mathrm{P}<0.01)$ by the administration of IBFF at three different doses, as shown in Fig. 2.

Effects of IBFF on the antioxidative status in D-Gal-induced liver injury. To determine whether IBFF attenuates the
A
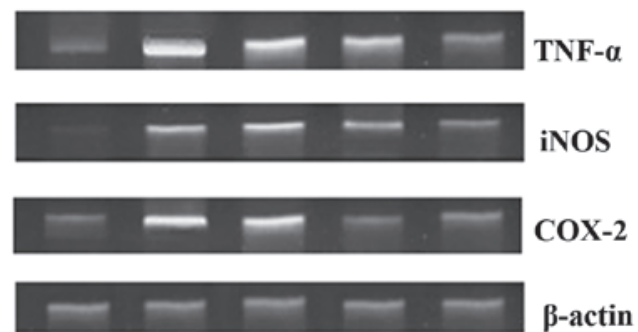

B

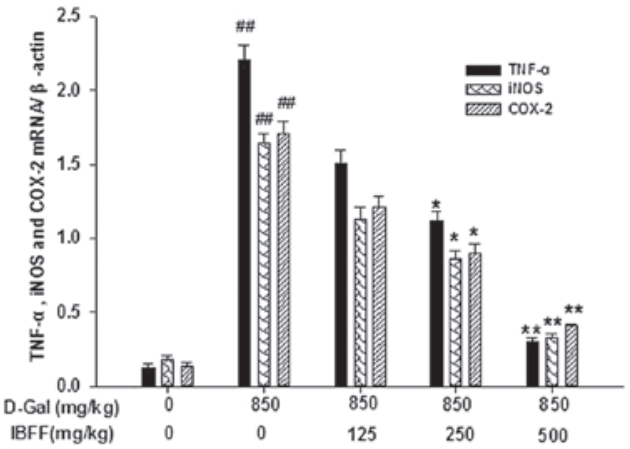

Figure 6. (A) Representative electrophoretic analysis of RT-PCR products. (B) Relative ratio of TNF- $\alpha$, iNOS and COX-2 densitometry to $\beta$-actin. ${ }^{\# \#} \mathrm{P}<0.01$ compared with the $\mathrm{D}$-Gal group. ${ }^{* *} \mathrm{P}<0.01,{ }^{*} \mathrm{P}<0.05$ compared with the control group. TNF- $\alpha$, tumor necrosis factor- $\alpha$; iNOS, inducible nitric oxide synthase; COX-2, cyclooxygenase- 2 .

increased oxidative damage in the livers of D-Gal-treated mice, we determined the levels of SOD, GSH-PX and CAT in the liver tissue. IBFF treatment inhibited the decrease in activity of antioxidant enzymes induced by D-Gal (Fig. 3).

Effect of IBFF on TNF- $\alpha, C O X-2$ and iNOS levels in $D$-Gal-induced liver injury. TNF- $\alpha$ and COX-2 are critical mediators of liver injury induced by D-Gal; injection of D-Gal induced the elevation of hepatic TNF- $\alpha$ and COX-2 levels. The serum TNF- $\alpha$ levels were $8.7 \pm 1.9 \mathrm{pg} / \mathrm{ml}$ in the control group. By contrast, the serum TNF- $\alpha$ levels were increased $\sim 4$-fold by $\mathrm{D}-\mathrm{Gal}$ and were attenuated by IBFF. The changes in the COX-2 and iNOS levels were similar to that of TNF- $\alpha$ (Fig. 4).

Effects of IBFF on histopathological changes in $D$-Gal-induced mouse liver injury. The liver tissues revealed multiple histopathological changes under an optical microscope (Fig. 5). Normal hepatic cells were arranged regularly and radially around the central vein, with an intact hepatic lobule structure. The liver cells of the mice treated with $850 \mathrm{ml} / \mathrm{kg}$ D-Gal appeared to have degenerated, the vacuoles underwent necrosis and an abnormal level of eosinophil infiltration existed in the portal tracts and liver lobules. After the administration of IBFF for 7 continuous days, the alleviation of degeneration and necrosis in the liver tissue was observed, accompanied by a reduction in the infiltration of inflammatory cells. The results of the histopathological evaluation revealed that IBFF exhibited a hepatoprotective effect against D-Gal-induced liver injury.

Effects of IBFF on TNF- $\alpha$, iNOS and COX-2 levels in $D$-Gal-induced mouse liver injury. Expression levels of hepatic TNF- $\alpha$, iNOS and COX-2 mRNA were low in the control group. However, the mRNA expression levels of these genes 
were increased by the administration of D-Gal. The expression of TNF- $\alpha$, iNOS and COX-2 mRNA was suppressed by all three doses of IBFF (Fig. 6).

\section{Discussion}

In this study, we investigated the protective effects of IBFF and its underlying mechanism on D-Gal-induced experimental liver injury in mice.

During hepatic injury, the deterioration of the integrity of the cellular membrane leads to the release of enzymes into the circulation, reflecting damage to the hepatic cells (12). Thus, abnormally high levels of serum hepatospecific enzymes, including ALT and AST, are considered to be good biomarkers for liver injury. We identified that IBFF inhibited the levels of ALT and AST in D-Gal-induced liver injury. The attenuated increase in serum ALT and AST levels suggests that IBFF improved the structural integrity of the hepatocellular membrane.

It has been demonstrated that oxidative stress is important in hepatotoxin-mediated liver injury and the same mechanism is involved in D-Gal-induced liver injury $(13,14)$. The level of MDA, which indicates the degree of lipid peroxidation, is an oxidative stress marker. In our study, IBFF inhibited the D-Gal-induced increase in MDA content in the mouse liver. Our findings suggest that IBFFs attenuate oxidative stress by decreasing the lipid peroxide level in the D-Gal-treated mouse liver.

The defense system of antioxidant enzymes includes SOD, CAT and GSH-PX. This study demonstrated that the activities of antioxidant enzymes in the mouse liver were markedly decreased by treatment with D-Gal. However, IBFF renewed the activities of the antioxidant enzymes in the livers of D-Gal-treated mice.

Furthermore, in addition to inducing direct cellular damage, oxidative stress activates the expression of various inflammatory genes implicated in hepatotoxicity. D-Gal activated monocytes to produce TNF- $\alpha$, a pro-inflammatory cytokine. It has been reported that increased levels of pro-inflammatory cytokines and neutrophils in the liver are associated with liver cell damage (15). TNF- $\alpha$ also stimulates the release of cytokines from macrophages and induces phagocyte oxidative metabolism and NO production. NO, a highly reactive oxidant produced through the action of iNOS, plays a role in a number of physiological processes $(16,17)$. This study confirmed an increase in the levels of TNF- $\alpha$ protein in the serum and mRNA expression in liver tissue after the intraperitoneal injection of D-Gal. These alterations were attenuated after pretreatment by oral administration of IBFF. IBFF also decreased the expression level of iNOS mRNA in liver tissue.

A number of studies have suggested that the NO released from inflammatory cells increases COX-2 activity (18). COX-2, which catalyzes the formation of prostaglandins and other eicosanoids from arachidonic acid, was induced at the site of inflammation. The production of prostanoids by COX-2 has often been implicated in inflammatory diseases characterized by edema and tissue injury (19). The present study demonstrated that IBFF attenuated the upregulation of COX-2 expression in the livers of D-Gal-treated mice, suggesting that IBFF attenuates inflammatory processes by suppressing the inflammatory gene expression.
In conclusion, IBFF inhibited the increase in AST and ALT levels, renewed the activities of antioxidant enzymes and attenuated the upregulation of TNF- $\alpha$, iNOS and COX-2 in the D-Gal-treated mouse liver. Our findings suggest that IBFF alleviates liver injury caused by D-Gal through antagonizing oxidative stress and the inflammatory response.

\section{References}

1. Malhi $\mathrm{H}$ and Gores GJ: Cellular and molecular mechanisms of liver injury. Gastroenterology 134: 1641-1654, 2008.

2. Ho SC, Liu JH and Wu RY: Establishment of the mimetic aging effect in mice caused by D-galactose. Biogerontology 4: 15-18, 2003.

3. Zhang X, Zhang A, Jiang B, Bao Y, Wang J and An L: Further pharmacological evidence of the neuroprotective effect of catalpol from Rehmannia glutinosa. Phytomedicine 15: 484-490, 2008.

4. Ramana BV, Kumar VV, Krishna PN, Kumar CS, Reddy PU and Raju TN: Effect of quercetin on galactose-induced hyperglycaemic oxidative stress in hepatic and neuronal tissues of Wistar rats. Acta Diabetol 43: 135-141, 2006.

5. Rafi MM, Bai NS, Chi-Tang-Ho, Rosen RT, White E, Perez D and Dipaola RS: A sesquiterpenelactone from Inula britannica induces anti-tumor effects dependent on Bcl-2 phosphorylation. Anticancer Res 25: 313-318, 2005.

6. Bai N, Lai CS, He K, Zhou Z, Zhang L, Quan Z, Zhu N, Zheng QY, Pan MH and Ho CT: Sesquiterpene lactones from Inula britannica and their cytotoxic and apoptotic effects on human cancer cell lines. J Nat Prod 69: 531-535, 2006.

7. Kobayashi T, Song QH, Hong T, Kitamura H and Cyong JC: Preventative effects of the flowers of Inula britannica on autoimmune diabetes in C57BL/KsJ mice induced by multiple low doses of streptozotocin. Phytother Res 16: 377-382, 2002.

8. Song QH, Kobayashi T, Hong T and Cyong JC: Effects of Inula Britannica on the production of antibodies and cytokines and on $\mathrm{T}$ cell differentiation in C57BL/6 mice immunized by ovalbumin. Am J Chin Med 30: 297-305, 2002.

9. Song QH, Kobayashi T, Iijima K, Hong T and Cyong JC: Hepatoprotective effects of Inula britannica on hepatic injury in mice. Phytother Res 14: 180-186, 2000.

10. Iijima K, Kiyohara H, Tanaka M, Matsumoto T, Cyong JC and Yamada $\mathrm{H}$ : Preventive effect of taraxasteryl acetate from Inula britannica subsp. japonica on experimental hepatitis in vivo. Planta Med 61: 50-53, 1995.

11. Zheng MS: An experimental study of the anti-HSV-II action of 500 herbal drugs. J Tradit Chin Med 9: 113-116, 1989.

12. Drotman RB and Lawhorn GT: Serum enzymes as indicators of chemically induced liver damage. Drug Chem Toxicol 1: 163-171, 1978.

13. Zhang ZF, Fan SH, Zheng YL, Lu J, Wu DM, Shan Q and $\mathrm{Hu}$ B: Purple sweet potato color attenuates oxidative stress and inflammatory response induced by d-galactose in mouse liver. Food Chem Toxicol 47: 496-501, 2009.

14. Zhang ZF, Fan SH, Zheng YL, Lu J, Wu DM, Shan Q and $\mathrm{Hu} \mathrm{B}$ : Troxerutin protects the mouse liver against oxidative stress-mediated injury induced by D-galactose. J Agric Food Chem 57: 7731-7736, 2009.

15. Hsieh CH, Frink M, Hsieh YC, Kan WH, Hsu JT, Schwacha MG, Choudhry MA and Chaudry IH: The role of MIP-1 alpha in the development of systemic inflammatory response and organ injury following trauma hemorrhage. J Immunol 181: 2806-2812, 2008.

16. Lowenstein CJ and Snyder SH: Nitric oxide, a novel biological messenger. Cell 70: 705-707, 1992.

17. Michel T and Feron O: Nitric oxide synthases: which, where, how, and why? J Clin Invest 100: 2146-2152, 1997.

18. Müller-Decker K, Berger I, Ackermann K, Ehemann V, Zoubova S, Aulmann S, Pyerin W and Fürstenberger G: Cystic duct dilatations and proliferative epithelial lesions in mouse mammary glands upon keratin 5 promoter-driven overexpression of cyclooxygenase-2. Am J Pathol 166: 575-584, 2005.

19. Tanaka Y, Takahashi M, Kawaguchi M and Amano F: Delayed release of prostaglandins from arachidonic acid and kinetic changes in prostaglandin $\mathrm{H}$ synthase activity on the induction of prostaglandin $\mathrm{H}$ synthase-2 after lipopolysaccharide-treatment of RAW264.7 macrophage-like cells. Biol Pharm Bull 20: 322-326, 1997. 